\title{
Higgs production at NNLO in QCD: the VBF channel
}

\author{
P. Bolzoni ${ }^{\mathrm{a}}{ }^{*}$, M. Zaro ${ }^{\text {b }}$, F. Maltoni ${ }^{\mathrm{b}}, \mathrm{S}$ Moch $^{\mathrm{a}}$ \\ ${ }^{a}$ Deutsches Elektronen-Synchrotron, DESY, \\ Platanenallee 6, D15738 Zeuthen, Germany \\ ${ }^{\mathrm{b}}$ Center for Particle Physics and Phenomenology (CP3), \\ Université Catholique de Louvain, \\ B-1348 Louvain-la-Neuve, Belgium
}

We present a phenomenological study for the production of the Higgs boson at next-to-next-to-leading order (NNLO) in QCD via the vector boson fusion (VBF) process. After a general discussion about the different production channels of the Higgs, we show results for hadron colliders like LHC and Tevatron in VBF. The theoretical predictions are obtained using the structure function approach. This approximation turns out to be more accurate than the precision to which the VBF Higgs production channel can be considered a well defined process by itself and the theoretical uncertainty are of the order of $1-2 \%$. The uncertainties due to parton distributions are also discussed and are estimated to be at the same level.

\section{INTRODUCTION}

To discover whether the Standard Model Higgs boson exists or not is one of the aims with most priority at the nowadays hadron colliders. These are the proton-anti-proton Tevatron machine at Fermilab and the proton-proton LHC machine at CERN. To achieve this goal an estimate of the expected events and the control over those processes which represent the background noise is required. Nevertheless in the case of a discovery of the Higgs it would become necessary to also investigate precisely its properties. On its turn this demands a determination of the cross section in the various production channels as precise as possible.

At hadron colliders the Higgs production channels which have a large enough cross section to be relevant are the gluon-gluon fusion Fig.1(a), the VBF Fig.1(b) and the associated production with $W, Z$ bosons and $t \bar{t}$ Fig.1(c,d).

Generally the dominant production mechanism is represented by the gluon fusion mediated by a top quark loop. For this production channel the first NNLO corrections in QCD have been com-

*Speaker

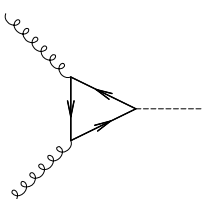

(a)

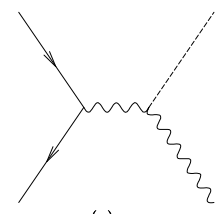

(c)

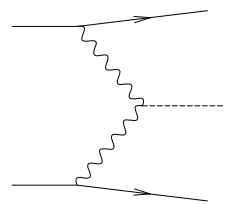

(b)

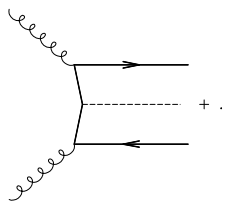

(d)
Figure 1. Higgs boson production channels at hadron colliders.

puted in the context of the effective theory in the limit large top quark mass limit [1-4]. It is also known that this approximation works very well up to the NNLO. Indeed the impact of the finite top mass effects are about a factor of ten smaller than the uncertainty due to the scale variation at the same order [5-7]. As far as the associated production of the Higgs with $W, Z$ bosons is concerned, the NNLO QCD corrections have been implemented in [8]. Thanks to these results the 
theoretical uncertainty is reduced to about $10 \%$ for the gluon fusion production mechanism and to less than a few percent for the associated production with gauge bosons. Very recently NNLO corrections in QCD have been included also for the VBF production mechanism [9] via the structure function approach thus reducing the theoretical uncertainty for this channel from the 5$10 \%$ of the NLO QCD and electroweak combined computations $[10,11]$ down to $1-2 \%$. This makes VBF the theoretically most accurate Higgs boson production channel at hadron colliders.

\section{THE VBF SIGNAL}

Among the production mechanisms of the Higgs boson that we have mentioned above, the VBF production mechanism can be considered by itself as a signal where its "background" includes also Higgs boson production by mechanisms other than VBF (see Fig.1).

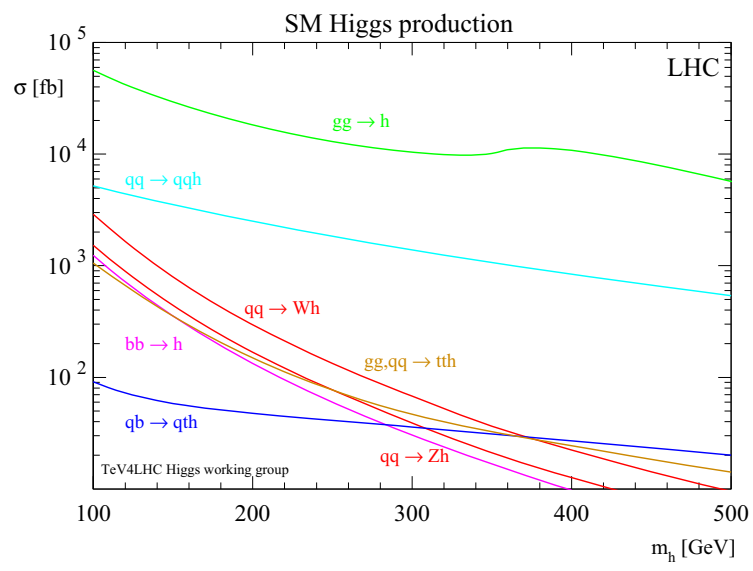

Figure 2. Higgs production cross sections at the LHC for the various channels (see Ref.[12] for more details about this plot).

Here we briefly describe its characteristics. Due to the preference of the Higgs to couple with heavy particles the VBF at LHC is second in size only to the gluon fusion channel which is mediated by a top quark loop (see Fig.2). Further- more it provides a clean experimental signature: it usually consists of two almost back to back hard tagging jets (generally with an invariant mass bigger than $600 \mathrm{GeV}$ and a pseudo-rapidity separation between them $\Delta \eta>4$ ) and the Higgs decay is confined in the central rapidity region. Imposing these constraints to the invariant mass and the rapidity of the jets as additional cuts (usually called VBF cuts) one reaches an impressive improvement of the signal-to-background ratio [13].

An heuristic way to qualitatively understand the property of the VBF signature is to consider angular ordering which arises from the dominant soft gluon emissions. One finds that the averaged azimuthal emission is confined to a cone limited by the angle between the emitter and the spectator parton. It can easily be shown that because of the behavior of the propagators in the $t$-channel in Fig.1(b) small values of the emission angle are favored. Because of this the hadronic activity in the VBF production mechanism is concentrated in the forward/backward region with respect to the incoming colored partons. This is in contrast to the gluon fusion channel where the radiation is expected and found in the central rapidity region [14].

Keeping in mind that what we strictly call VBF signal is the Higgs boson weak production with a color singlet exchange in the $t$-channel, we want now to discuss the possible interference effects with other processes. Already at LO the VBF process in Fig.1(b) can interfere with the production mechanism in Fig.1(c) where the associated produced gauge boson decays into two quarks. However as it is shown in [11] this interference effect is at the per mil level. At higher orders, interference effects occur with the gluon fusion production mechanism in Fig.1. Also in this case the interference effect is small $[15,16]$ and it turns out to be well below the percent level. This demonstrates that the Higgs boson production via the VBF mechanism can be defined a signal by itself within an ambiguity better than the $1 \%$. It is this ambiguity in defining the Higgs boson production via $\mathrm{VBF}$ that sets also the target theoretical precision for this observable. 


\section{THE COMPUTATION}

Here we want to illustrate the structure function approach in the VBF production chnnel [10] and show that this approximation remains sufficiently accurate even at NNLO in QCD.

The structure function approach consists basically in viewing the VBF process as a double deep-inelastic scattering (DIS) attached to the colorless pure electroweak vector boson fusion into a Higgs boson. A qualitative illustration of the structure function approach is given in Fig.3. According to this approach one can include NLO QCD corrections to the VBF process employing the standard DIS structure functions $F_{i}\left(x, Q^{2}\right) ; i=1,2,3$ at NLO [17]. Similarly at the NNLO level one has to employ the corresponding structure functions [18-21].

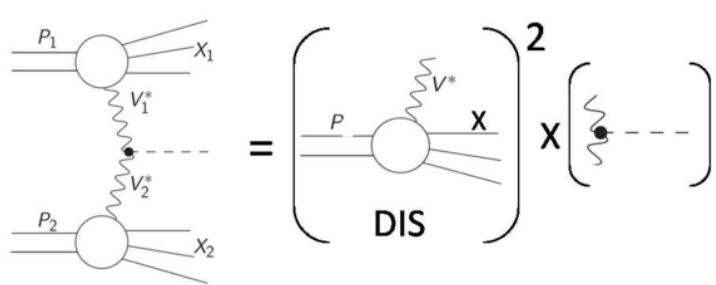

Figure 3. Schematic view of the structure function approach.

The structure function approach represents a very accurate approximation because it is based on the absence or smallness of the QCD interference contributions between the two inclusive final states $X_{1}$ and $X_{2}$. We now discuss the various contributions up to NNLO which in principle violate the structure function approach but which can nevertheless be safely neglected.

At LO there is already a structure function violating contribution coming from the interferences between identical final state quarks (e.g. $u u \rightarrow H u u)$ or between processes where either a $W$ or a $Z$ can be exchanged (e.g. $u d \rightarrow H u d$ ). Simple kinematical arguments show that such contributions are very small and contribute to the total cross section well below the percent level [22]. These contributions can be easily computed and have been included in our results anyway.

At the NLO level possible contributions violating the structure function approach arise when a gluon in the $t$-channel is exchanged between the upper and the lower quark line in Fig.2(b). However the interference of such one-loop contributions with the LO diagram have a vanishing color factor due to the generators $t^{a}\left(a=1, \ldots, N_{c}^{2}-1\right)$ of the color group $S U\left(N_{c}\right)$ being traceless. This means that apart from the interference effects discussed at LO the structure function approach represents an exact approach to the computation.

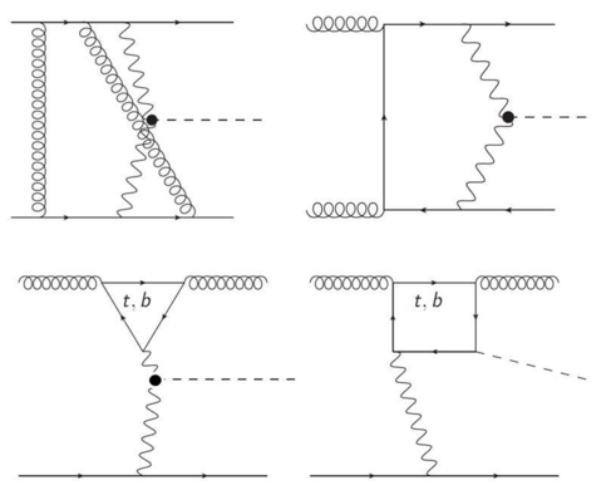

Figure 4. Examples of neglected Feynman diagrams at NNLO.

At NNLO the structure function approach is not exact but it can be still considered a very good approximation. The types of diagrams that violate the structure function approach are shown in Fig.4. The first type represents a double gluonexchange in the $t$-channel (note that one or both of the two emissions could also be real); the second type is a representative of the so-called single quark line (SQL) diagrams contributing at NNLO; the last two are heavy quarks (top and bottom) loop diagrams. The first type of contributions represents a gauge invariant, infrared and ultraviolet finite class of diagrams. Another 


\begin{tabular}{llll}
\hline$m_{h}(\mathrm{GeV})$ & \multicolumn{1}{c}{120} & \multicolumn{1}{c}{300} & \multicolumn{1}{c}{500} \\
\hline $1.96 \mathrm{TeV}$ & $3.87 \mathrm{E}-6(0.0690)$ & $2.52 \mathrm{E}-7(0.0054)$ & $1.50 \mathrm{E}-8(0.00042)$ \\
$7 \mathrm{TeV}$ & $2.62 \mathrm{E}-4(1.235)$ & $7.89 \mathrm{E}-5(0.614)$ & $2.73 \mathrm{E}-5(0.088)$ \\
\hline
\end{tabular}

Table 1

Total cross section (pb) from the neglected triangle diagram in Fig.4. In parenthesis are reported also the numbers included in the NNLO computation in the structure function approach employing the MSTW PDF set [26]. All the parameters have been taken from PDG 2008 [27].

characteristic of this class of diagrams is its color suppression by a factor of $1 / N_{c}^{2}$ with respect to the contributions included by the structure function approach. Furthermore this type of contributions are also strongly kinematically suppressed [23-25]. This is mainly due to the behavior of the gluon propagator in the $t$-channel and/or to the small overlapping of the phase space of real emissions from the upper quark line and real emissions from the lower one. The neglected SQL type contributions in Fig.4 do not represent a class of infrared safe diagrams. However as shown in [13] their impact is small enough not to produce a significant deterioration of the VBF signal. Also, these color exchange effects are, by our definition, no VBF processes. Finally we take into consideration the triangle and the box contributions in Fig.4. Even if a full computation is in progress [28] as a first rough estimation we have computed the triangle contribution in Fig.4 in the limit of infinite top mass. In Table 1 we report some values of the contribution to the total cross section from the triangle at $1.96 \mathrm{TeV}$ for the Tevatron and at $7 \mathrm{TeV}$ for the LHC. This has been checked performing two independent computations. As we can see from Table 1 its impact is very small and can be safely neglected.

\section{RESULTS AT HADRON COLLIDERS}

Here we discuss some numerical results obtained for the $\mathrm{VBF}$ production mechanism at hadron colliders up to the NNLO in QCD employing the structure function approach.

For the electroweak parameters we have used the central values released by the PDG collaboration in 2008 [27] while for the parton distributions functions we choose the set MSTW [26]. For

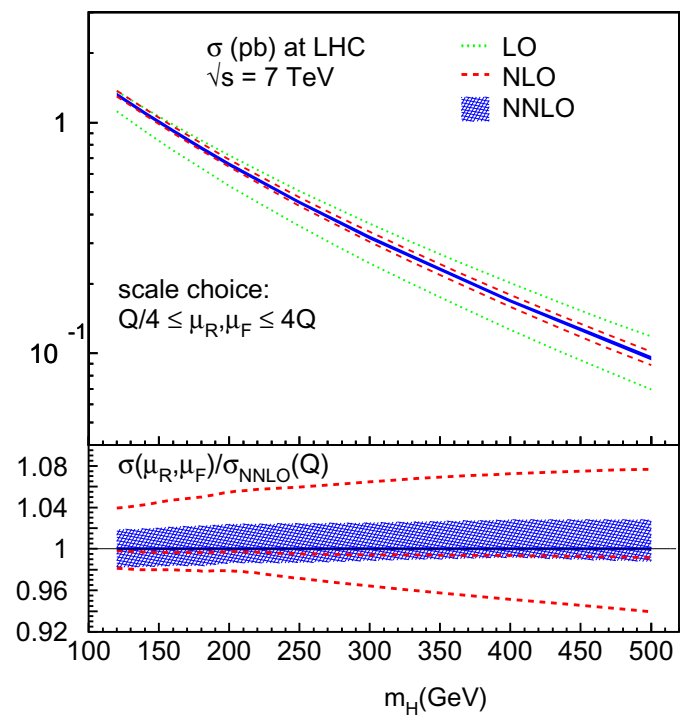

Figure 5. Total cross section for the LHC at $7 \mathrm{TeV}$ employing the MSTW PDF set [26].

the LHC we set the center of mass energy to be $\sqrt{S}=7 \mathrm{TeV}$ for the LHC and $\sqrt{S}=1.96 \mathrm{TeV}$ for the Tevatron.

In Fig. 5 we plot the total cross section for the VBF production mechanism at the $\mathrm{LHC}$ at $7 \mathrm{TeV}$. The LO, NLO and NNLO results in QCD are shown as a function of the Higgs boson mass which first of all induce only a rather mildly dependence on it. The bands represent the theoretical uncertainty of the prediction. They have been obtained varying the factorization and the renormalization scales in the quite large range $\mu_{\mathrm{R}(\mathrm{F})} \in[Q / 4,4 Q]$ where $Q$ is the virtuality of the vector bosons which "fuse" into the Higgs. Clearly other scale choices are possible (e.g. the 


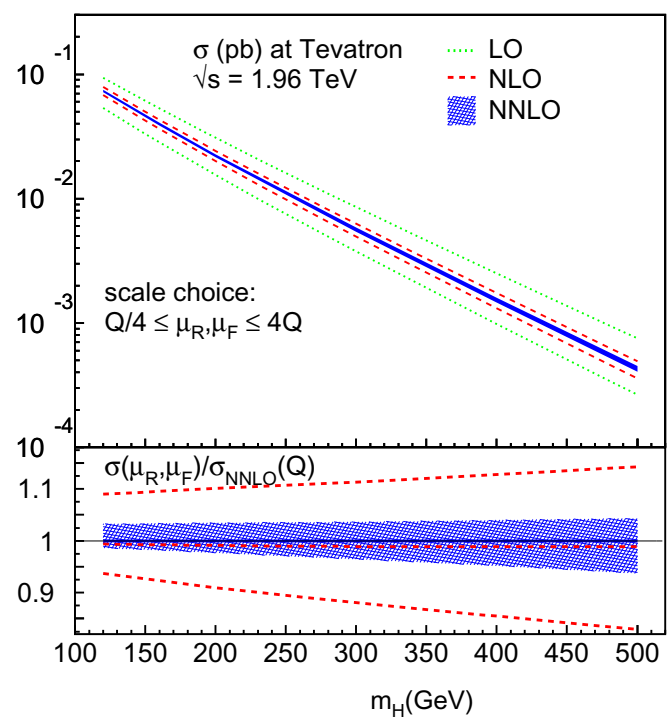

Figure 6. The same as Fig.5 for the Tevatron.

choice $Q=m_{h}$ the Higgs mass) but the one chosen for the plot turned out to be the more natural choice because it exhibits a better convergence of the perturbative expansion. This also shows that at NNLO in QCD the theoretical uncertainty is reduced to be less than the $2 \%$ reaching the same level of ambiguity at which the Higgs production signal via $\mathrm{VBF}$ can be defined phenomenologically.

In Fig.6 we report on numbers for the Tevatron where the center of mass energy is set to $1.96 \mathrm{TeV}$. As one can expect the total cross section shows the same behavior upon varying the Higgs mass and it is almost an order of magnitude smaller. Again the lower part of Fig. 5 shows a very good convergence of the perturbative QCD expansion. Even if the theoretical uncertainty remains slightly bigger compared to the LHC the relative improvement with respect to the NLO prediction is at the same order of percentage.

Finally we consider also the uncertainties coming from the parton distributions. To achieve this we have employed the MSTW $68 \%$ confidence level PDF sets [26] and compare with other NNLO PDF sets, i.e. ABKM [29] and JR09VF [30]. The results in Figs.7, 8 show that an almost

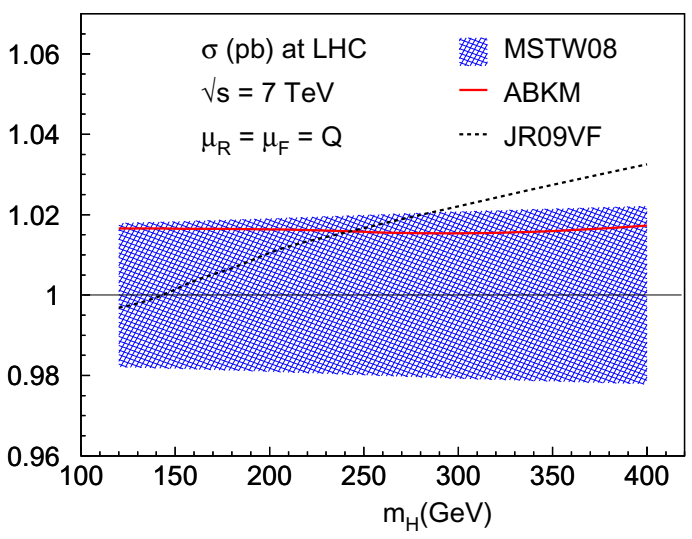

Figure 7. The PDF uncertainty of the total cross section at NNLO for $\mathrm{LHC}$ at $7 \mathrm{TeV}$ for the $68 \%$ CL MSTW PDF set [26]. For ABKM [29] and JR09VF [30] the ration of the central value is plotted.

constant 2\% PDF uncertainty can be associated to the cross section for both the LHC and the Tevatron. In the case of the Tevatron the difference between the MSTW, ABKM and JR sets is due to larger uncertainties for the high- $x$ quark PDFs [29].

To conclude we give the address of the web interface where our code for the NNLO VBF total cross section can be used online [31]. After the registration, setting on a dialog window the energy and choosing the hadron collider the numerical answer is received per email.

\section{CONCLUSIONS}

We have described recent progress for the VBF Higgs predictions and investigated at which level it can be considered a well defined process by itself. Then after showing how well the structure function approach works even at the NNLO in QCD we have employed it to obtain predictions at the hadron colliders. The theoretical uncertainty is estimated to be less than $2 \%$ which is compatible with the ambiguity at which this signal can be defined. Finally what has been presented here is a natural first step towards less inclusive (e.g. rapidity distributions) predictions of this process 


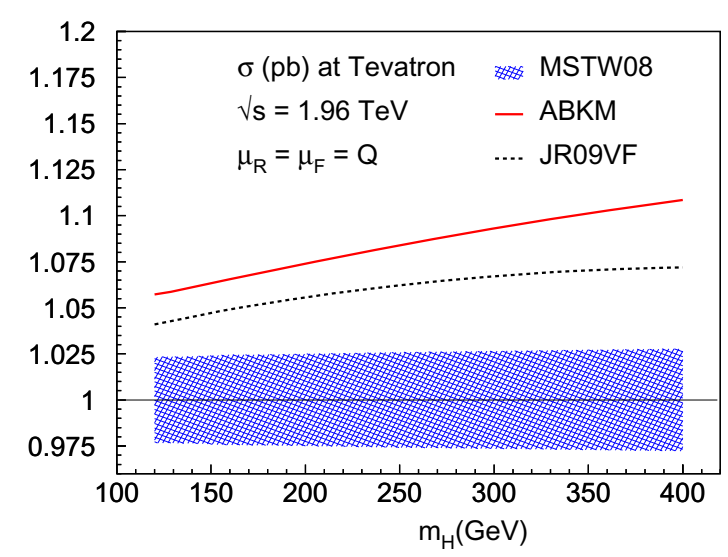

Figure 8. Same as Fig.7 for the Tevatron.

at NNLO in QCD.

\section{REFERENCES}

1. R. V. Harlander, Phys. Lett. B492, 74 (2000), arXiv:hep-ph/0007289.

2. C. Anastasiou and K. Melnikov, Nucl. Phys. B646, 220 (2002), arXiv:hep-ph/0207004.

3. M. Steinhauser, Phys. Rept. 364, 247 (2002), arXiv:hep-ph/0201075.

4. S. Catani, D. de Florian, M. Grazzini, and P. Nason, JHEP 07, 028 (2003), arXiv:hep$\mathrm{ph} / 0306211$.

5. R. V. Harlander, H. Mantler, S. Marzani, and K. J. Ozeren, Eur. Phys. J. C66, 359 (2010), arXiv:0912.2104.

6. S. Marzani, R. D. Ball, V. Del Duca, S. Forte, and A. Vicini, Nucl. Phys. B800, 127 (2008), arXiv:0801.2544.

7. A. Pak, M. Rogal, and M. Steinhauser, JHEP 02, 025 (2010), arXiv:0911.4662.

8. O. Brein, A. Djouadi, and R. Harlander, Phys. Lett. B579, 149 (2004), arXiv:hepph/0307206.

9. P. Bolzoni, F. Maltoni, S.-O. Moch, and M. Zaro, (2010), arXiv:1003.4451.

10. T. Han, G. Valencia, and S. Willenbrock, Phys. Rev. Lett. 69, 3274 (1992), arXiv:hepph/9206246.

11. M. Ciccolini, A. Denner, and S. Dittmaier, Phys. Rev. D77, 013002 (2008),
arXiv:0710.4749.

12. T. Hahn, S. Heinemeyer, F. Maltoni, G. Weiglein, and S. Willenbrock, (2006), arXiv:hepph/0607308.

13. R. V. Harlander, J. Vollinga, and M. M. Weber, Phys. Rev. D77, 053010 (2008), arXiv:0801.3355.

14. V. Del Duca, A. Frizzo, and F. Maltoni, JHEP 05, 064 (2004), arXiv:hep-ph/0404013.

15. J. R. Andersen and J. M. Smillie, Phys. Rev. D75, 037301 (2007), arXiv:hep-ph/0611281.

16. J. R. Andersen, T. Binoth, G. Heinrich, and J. M. Smillie, JHEP 02, 057 (2008), arXiv:0709.3513.

17. W. A. Bardeen, A. J. Buras, D. W. Duke, and T. Muta, Phys. Rev. D18, 3998 (1978).

18. D. I. Kazakov, A. V. Kotikov, G. Parente, O. A. Sampayo, and J. Sanchez Guillen, Phys. Rev. Lett. 65, 1535 (1990).

19. E. B. Zijlstra and W. L. van Neerven, Phys. Lett. B297, 377 (1992).

20. E. B. Zijlstra and W. L. van Neerven, Nucl. Phys. B383, 525 (1992).

21. S. Moch and J. A. M. Vermaseren, Nucl. Phys. B573, 853 (2000), arXiv:hep$\mathrm{ph} / 9912355$.

22. D. A. Dicus and S. S. D. Willenbrock, Phys. Rev. D32, 1642 (1985).

23. W. L. van Neerven and J. A. M. Vermaseren, Phys. Lett. B142, 80 (1984).

24. J. Blumlein, G. J. van Oldenborgh, and R. Ruckl, Nucl. Phys. B395, 35 (1993), arXiv:hep-ph/9209219.

25. T. Figy, V. Hankele, and D. Zeppenfeld, JHEP 02, 076 (2008), arXiv:0710.5621.

26. A. D. Martin, W. J. Stirling, R. S. Thorne, and G. Watt, Eur. Phys. J. C63, 189 (2009), arXiv:0901.0002.

27. Particle Data Group, C. Amsler et al., Phys. Lett. B667, 1 (2008).

28. P. Bolzoni, F. Maltoni, S.-O. Moch, and M. Zaro, (in preparation).

29. S. Alekhin, J. Blumlein, S. Klein, and S. Moch, Phys. Rev. D81, 014032 (2010), arXiv:0908.2766.

30. P. Jimenez-Delgado and E. Reya, Phys. Rev. D80, 114011 (2009), arXiv:0909.1711.

31. http://vbf-nnlo.phys.ucl.ac.be/vbf .html. 